\title{
Direct formulation of the supersonic acoustic intensity in space domain
}

\author{
Fernandez Grande, Efren; Jacobsen, Finn; Leclre, Quentin
}

Published in:

Journal of the Acoustical Society of America

Link to article, DOI:

$10.1121 / 1.3662052$

Publication date:

2012

Document Version

Publisher's PDF, also known as Version of record

Link back to DTU Orbit

Citation (APA):

Fernandez Grande, E., Jacobsen, F., \& Leclre, Q. (2012). Direct formulation of the supersonic acoustic intensity in space domain. Journal of the Acoustical Society of America, 131(1), 186-193.

https://doi.org/10.1121/1.3662052

\section{General rights}

Copyright and moral rights for the publications made accessible in the public portal are retained by the authors and/or other copyright owners and it is a condition of accessing publications that users recognise and abide by the legal requirements associated with these rights.

- Users may download and print one copy of any publication from the public portal for the purpose of private study or research.

- You may not further distribute the material or use it for any profit-making activity or commercial gain

- You may freely distribute the URL identifying the publication in the public portal

If you believe that this document breaches copyright please contact us providing details, and we will remove access to the work immediately and investigate your claim. 


\title{
Direct formulation of the supersonic acoustic intensity in space domain
}

\author{
Efren Fernandez-Grande ${ }^{\text {a) }}$ and Finn Jacobsen \\ Acoustic Technology, Department of Electrical Engineering, Technical University of Denmark (DTU), \\ Building 352, Ørsteds Plads, DK-2800 Kongens Lyngby, Denmark \\ Quentin Leclère \\ Laboratoire Vibrations Acoustique, INSA Lyon, F-69621 Villeurbanne Cedex, France
}

(Received 19 July 2011; revised 7 October 2011; accepted 17 October 2011)

\begin{abstract}
This paper proposes and examines a direct formulation in space domain of the so-called supersonic acoustic intensity. This quantity differs from the usual (active) intensity by excluding the circulating energy in the near-field of the source, providing a map of the acoustic energy that is radiated into the far field. To date, its calculation has been formulated in the wave number domain, filtering out the evanescent waves outside the radiation circle and reconstructing the acoustic field with only the propagating waves. In this study, the supersonic intensity is calculated directly in space domain by means of a two-dimensional convolution between the acoustic field and a spatial filter mask that corresponds to the space domain representation of the radiation circle. Therefore, the acoustic field that propagates effectively to the far field is calculated via direct filtering in space domain. This paper presents the theory, as well as a numerical example to illustrate some fundamental principles. An experimental study on planar radiators was conducted to verify the validity of the technique. The experimental results are presented, and serve to illustrate the usefulness of the analysis, its strengths and limitations. (C) 2012 Acoustical Society of America. [DOI: 10.1121/1.3662052]
\end{abstract}

PACS number(s): 43.40.Rj, 43.60.Sx, 43.20.Rz, 43.20.Ye [EGW]

Pages: 186-193

\section{INTRODUCTION}

The concept of efficient radiation is central in the analysis of sound radiation from plates and other wave bearing structures, because it gives an indication of the flow of acoustic energy that is radiated effectively into the far field. It indicates how much of the acoustic energy flow in the near field of a source is energy that propagates to the far field, and how much is circulating energy resulting from the interaction of propagating and evanescent waves near the source.

The far field radiation of sources is often the quantity of concern in acoustics and noise control, because this is the quantity to which a potential observer is typically exposed. Consequently, it has been studied extensively in the literature for decades, e.g., sound radiation from plates and panels, ${ }^{1-3}$ identification of velocity patterns that radiate effectively into the far field based on singular value analysis, ${ }^{4-6}$ etc.

The wave number processing of sound fields and the development of near-field acoustic holography (NAH), ${ }^{7,8}$ have laid a new ground for calculating in practice the efficient and inefficient radiation from sound sources. Williams ${ }^{9,10}$ introduced the concept of supersonic acoustic intensity for identifying and characterizing the regions of a source that radiate effectively into the far field.

The term "supersonic intensity" was chosen because of its close connection with the supersonic flexural waves in a structure. ${ }^{10}$ However, this terminology might lead to the notion that the acoustic waves related to this quantity are

\footnotetext{
a) Author to whom correspondence should be addressed. Electronic mail: efg@elektro.dtu.dk
}

supersonic, although this is not the case, their propagation speed is perfectly sonic.

So far, the calculation of the supersonic intensity has been formulated in the framework of Fourier based NAH, where the sound field is explicitly transformed into the wave number domain. The wave number components outside the radiation circle, ${ }^{8}$ which correspond to evanescent waves, are filtered out, and the reconstruction is based only on the terms inside the radiation circle, which correspond to the waves that propagate to the far field.

The purpose of this paper is to propose and examine a formulation of the supersonic intensity directly in the space domain. The calculation is expressed as a two-dimensional convolution product between the acoustic field and a spatial operator or filter mask. The implementation of the method is straightforward and does not require transformations into the wave number domain. This approach can be convenient for estimating the efficient radiation of a source when the Fourier transformation of the sound field is not otherwise required. This is the case, for instance, when using holographic methods that are not based on FFT processing, ${ }^{11-14}$ applied directly to measured data, etc.

The paper presents the theory and provides a numerical illustration of a one-dimensional radiator, revisiting the concept of effective and ineffective radiation. An experimental study is also included, which aims at illustrating the usefulness of the supersonic acoustic intensity in practice.

\section{THEORY}

\section{A. Fourier based supersonic acoustic intensity}

Consider the wave number spectra of the pressure and normal component of the particle velocity in a plane $z:{ }^{8}$ 


$$
\begin{aligned}
& P\left(k_{x}, k_{y}, z\right)=\iint_{-\infty}^{\infty} p(x, y, z) e^{j\left(k_{x} x+k_{y} y\right)} d x d y, \\
& U\left(k_{x}, k_{y}, z\right)=\iint_{-\infty}^{\infty} u(x, y, z) e^{j\left(k_{x} x+k_{y} y\right)} d x d y,
\end{aligned}
$$

where the time dependence $e^{j \omega t}$ is omitted. The sound pressure and particle velocity that are radiated into the far field are associated with the spectral energy inside the radiation circle,

$$
\begin{aligned}
& p^{(s)}(x, y, z)=\frac{1}{4 \pi^{2}} \iint_{S r} P\left(k_{x}, k_{y}, z\right) e^{-j\left(k_{x} x+k_{y} y\right)} d k_{x} d k_{y}, \\
& u^{(s)}(x, y, z)=\frac{1}{4 \pi^{2}} \iint_{S r} U\left(k_{x}, k_{y}, z\right) e^{-j\left(k_{x} x+k_{y} y\right)} d k_{x} d k_{y}
\end{aligned}
$$

where $S r$ denotes the surface corresponding to the radiation circle. The flow of acoustic energy that is radiated effectively into the far field, i.e., the supersonic intensity, is defined as

$$
I^{(s)}(x, y, z)=\frac{1}{2} \operatorname{Re}\left\{p^{(s)}(x, y, z) u^{(s)}(x, y, z)^{*}\right\}
$$

where the subscript asterisk denotes the complex conjugate.

It is apparent that the supersonic intensity is essentially a spatially low-pass filtered version of the conventional active intensity, where the evanescent waves are filtered out.

\section{B. Direct formulation in space domain}

Let the function $H\left(k_{x}, k_{y}\right)^{(s)}$ be defined as the unit circle function,

$$
H\left(k_{x}, k_{y}\right)^{(s)}= \begin{cases}1 & \text { if }\left(k_{x}^{2}+k_{y}^{2}\right)<k^{2} \\ \frac{1}{2} & \text { if }\left(k_{x}^{2}+k_{y}^{2}\right)=k^{2} \\ 0 & \text { if }\left(k_{x}^{2}+k_{y}^{2}\right)>k^{2}\end{cases}
$$

which represents a two-dimensional circular unit pulse, with the transition from zero to one at the boundary of the radiation circle.

The pressure and velocity that are radiated into the far field, Eqs. (3) and (4), can be expressed as

$$
\begin{aligned}
p^{(s)}(x, y, z)= & \iint_{-\infty}^{\infty} P\left(k_{x}, k_{y}, z\right) \cdot H^{(s)}\left(k_{x}, k_{y}\right) \\
& \times e^{-j\left(k_{x} x+k_{y} y\right)} d k_{x} d k_{y}, \\
u^{(s)}(x, y, z)= & \iint_{-\infty}^{\infty} U\left(k_{x}, k_{y}, z\right) \cdot H^{(s)}\left(k_{x}, k_{y}\right) \\
& \times e^{-j\left(k_{x} x+k_{y} y\right)} d k_{x} d k_{y} .
\end{aligned}
$$

From the convolution theorem, the products in the wave number domain in Eqs. (7) and (8) are equivalent in space domain to a two-dimensional convolution between the acoustic field and $h^{(s)}(x, y)$,

$$
\begin{aligned}
& p^{(s)}(x, y, z)=p(x, y, z) * h^{(s)}(x, y), \\
& u^{(s)}(x, y, z)=u(x, y, z) * h^{(s)}(x, y) .
\end{aligned}
$$

The two-dimensional convolution is defined as

$$
p^{(s)}(x, y, z)=\iint_{-\infty}^{\infty} p\left(x^{\prime}, y^{\prime}, z\right) h^{(s)}\left(x-x^{\prime}, y-y^{\prime}\right) d x^{\prime} d y^{\prime} .
$$

Note that the function $h^{(s)}(x, y)$ is the space domain version of the radiation circle. Because of its relation with the radiation circle, it will be referred to as the radiation filter mask or radiation kernel. It can be calculated by inverse transformation of the function $H^{(s)}\left(k_{x}, k_{y}\right)$ from the wave number domain to the space domain,

$$
h^{(s)}(x, y)=\frac{1}{4 \pi^{2}} \iint_{-\infty}^{\infty} H^{(s)}\left(k_{x}, k_{y}\right) e^{-j\left(k_{x} x+k_{y} y\right)} d k_{x} d k_{y} .
$$

Due to the geometry and properties of $H^{(s)}\left(k_{x}, k_{y}\right)$, it is convenient to introduce polar coordinates, so that $\rho=\sqrt{x^{2}+y^{2}}$ and $k_{\rho}=\sqrt{k_{x}^{2}+k_{y}^{2}}$. The function $H^{(s)}\left(k_{\rho}\right)$ is defined in polar coordinates as

$$
H^{(s)}\left(k_{\rho}\right)= \begin{cases}1 & \text { if } k_{\rho}<k \\ \frac{1}{2} & \text { if } k_{\rho}=k \\ 0 & \text { if } k_{\rho}>k\end{cases}
$$

Because $H^{(s)}\left(k_{\rho}\right)$ is circularly symmetric, the inverse Fourier transform of Eq. (12) can be expressed as an inverse Hankel transform,

$$
h^{(s)}(\rho)=\frac{1}{2 \pi} \int_{0}^{\infty} H_{k}^{(s)}\left(k_{\rho}\right) J_{0}\left(k_{\rho} \rho\right) k_{\rho} d k_{\rho} .
$$

The $H_{k}^{(s)}\left(k_{\rho}\right)$ function is zero for $k_{\rho}>k$. Therefore, the previous integral is equivalent to

$$
h^{(s)}(\rho)=\frac{1}{2 \pi} \int_{0}^{k} J_{0}\left(k_{\rho} \rho\right) k_{\rho} d k_{\rho} .
$$

This integral can be evaluated analytically making use of the relation $\int x J_{0}(a x) d x=(x / a) J_{1}(a x),{ }^{15}$ and therefore, the radiation filter mask is

$$
h^{(s)}(\rho)=\frac{k}{2 \pi \rho} J_{1}(k \rho),
$$

which back in rectangular coordinates is

$$
h^{(s)}(x, y)=\frac{k}{2 \pi \sqrt{x^{2}+y^{2}}} J_{1}\left(k \sqrt{x^{2}+y^{2}}\right) .
$$

This function is shown in Fig. 1. 

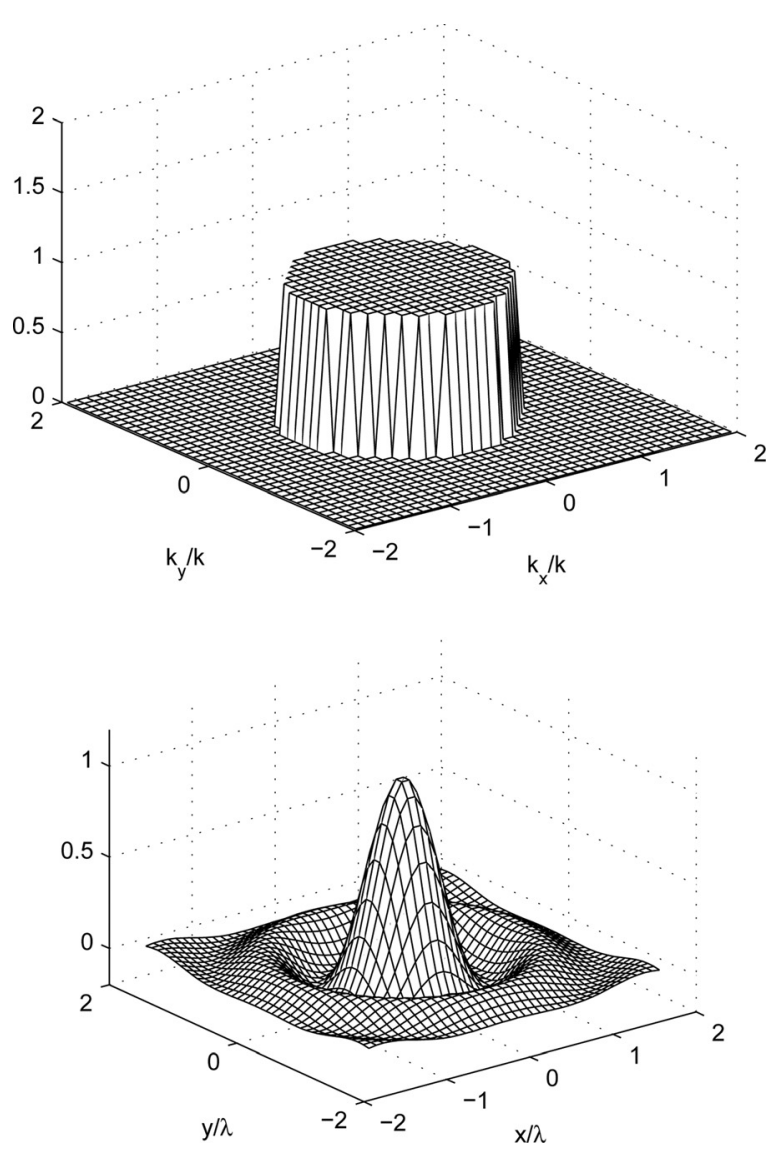

FIG. 1. (Top) Radiation filter in the wave number domain $H^{(s)}\left(k_{x}, k_{y}\right)$ and (bottom) the corresponding radiation kernel in the space domain $h^{(s)}(x, y)$. The axes are normalized. Note that the width of the mainlobe of $h^{(s)}$ is equal to the wavelength in air.

Using Eq. (17) for $h^{(s)}(x, y)$, the supersonic intensity can easily be calculated from Eqs. (5), (9), and (10)

Consider the example of a baffled monopole with volume velocity $Q$ radiating into free field half-space. In the source plane $(z=0)$, the particle velocity component normal to the $x, y$ plane is $u_{z}(x, y, 0)=Q \delta(x) \delta(y)$. From Eq. (10) and the shifting property of the Dirac delta function, it follows that the supersonic normal velocity of the baffled point source is

$$
u_{z}^{(s)}(x, y, 0)=\frac{k Q}{2 \pi \rho} J_{1}(k \rho)
$$

This expression is identical to the expression derived in Ref. 10.

\section{Discrete formulation}

Consider a square uniform array of dimensions $N \times N$ and a radiation kernel of $L \times L$. The general left justified form of the discrete two-dimensional convolution is

$$
\begin{aligned}
p^{(s)}(x, y, z)= & H_{0} \sum_{m=0}^{N-1} \sum_{n=0}^{N-1} p\left(x_{m}^{\prime}, y_{n}^{\prime}, z\right) \\
& \times h^{(s)}\left(x-x_{m}^{\prime}, y-y_{n}^{\prime}\right),
\end{aligned}
$$

where $H_{0}$ corresponds to the area of each grid position, $H_{0}=\Delta x \Delta y$. Note that due to the circular symmetry of the filter mask, the convolution product is equivalent to a twodimensional correlation. The output of the convolution is of dimensions $M \times M$, where $M=N+L-1$.

The convolution sum can be formulated in vector-space as

$$
\boldsymbol{p}_{\left[M^{2} \times 1\right]}^{(s)}=\boldsymbol{T}_{\left[M^{2} \times N^{2}\right]} \cdot \boldsymbol{p}_{\left[N^{2} \times 1\right]},
$$

where $\boldsymbol{T}$ is a $M^{2} \times N^{2}$ matrix containing the "shifting" radiation filter mask. Each column of $\boldsymbol{T}$ operates on a single point of the input matrix, and it accounts for the shifting of the spatial filter mask through that point. For details of the implementation see Refs. 16 and 17.

\section{ONE-DIMENSIONAL RADIATOR}

It is interesting to consider a one-dimensional finite radiator because it is a simple illustration and yet the generalization to the two-dimensional case is straightforward. The onedimensional supersonic filter in the wave number domain is ideally a rectangular step function with a cutoff frequency determined by the wave number in air, $k$. The inverse Fourier transform of this filter is the radiation kernel in one dimension,

$$
h_{1 D}^{(s)}(x)=A_{h} \operatorname{sinc}(k x),
$$

with $A_{h}=2 k$. It follows from this expression that the filter has an infinite impulse response in space domain, due to the ideal cut-off. This infinitely long response is not well compatible with a finite measurement aperture. Furthermore, it is a well-known fact that the ideal low-pass filtering introduces unwanted ringing artifacts via the Gibbs phenomenon. Therefore, it is convenient to define a finite filter mask that is more suited in practice to the non-ideal case. A simple and well established solution in signal processing and imaging to minimize ringing artifacts and preserve the general profile of the ideal filter is to use a Lanczos filter, ${ }^{18,19}$

$$
h_{w, 1 D}^{(s)}(x)= \begin{cases}A_{h} \operatorname{sinc}(k x) \operatorname{sinc}(k x / a), & \text { for }-a<k x<a \\ 0 & \text { otherwise }\end{cases}
$$

where $a$ determines how many sidelobes of the ideal filter are included before tapering to zero. This filter can be seen as an ideal brick-wall filter, weighted with the mainlobe of a sinc function. Typical values of $a$ are $a=[1,2,3, \ldots]$, because integer numbers make the two sincs of Eq. (22) be zero at the edge of the filter mask, providing a smooth cut-off.

The source considered is a theoretical one-dimensional plate, $4 \mathrm{~m}$ long and simply supported at the boundaries. Figures 2(a) and 2(b) show the two radiation filter masks from Eqs. (21) and (22) for a wave number in air of $k=5 \mathrm{rad} / \mathrm{m}$. Figures 2(c) and 2(d) show the normal velocity profile of the 1-D radiator in its $m=5$ and $m=9$ modes, respectively. Figures 2(e) and 2(f) show the result of the direct convolution between the two modes and the radiation filter masks. The spatial wave number of the $m=5$ mode is $k_{x}=m \pi / L \approx 4 \mathrm{rad} / \mathrm{m}$. In this case, the main-lobe width of the supersonic operator, $\sim 1.25 \mathrm{~m}$, is smaller than the spatial wavelength of the plate, 

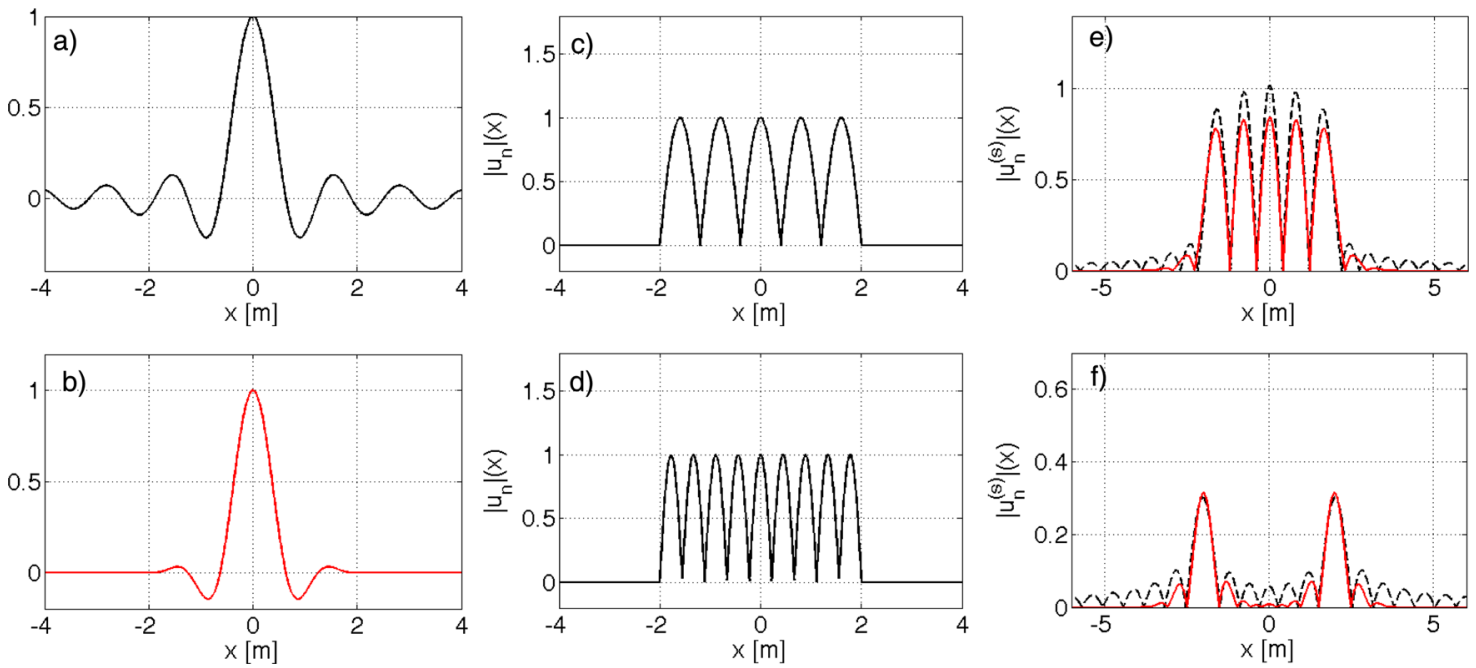

FIG. 2. (Color online) One-dimensional radiator above and below coincidence. (a) Wave number in air $k=5 \mathrm{rad} / \mathrm{m}$ : Ideal radiation filter mask, as in Eq. (21). (b) Finite radiation filter mask, as in Eq. (22) with $a=3$. Normal velocity profile of the radiator (c) for an $m=5$ mode and (d) for an $m=9$ mode. Result of the convolution with the filter mask (e) for the $m=5$ mode and (f) for the $m=9$ mode, with the ideal filter (dashed line) and with the finite filter (solid line). The ordinate axes are normalized.

$1.6 \mathrm{~m}$, and all regions contribute efficiently to the far field radiation. For the $m=9$ mode, the spatial wave number is $k_{x} \approx 7 \mathrm{rad} / \mathrm{m}$ (spatial wavelength of $0.8 \mathrm{~m}$ ), and the plate does not radiate efficiently because neighboring regions cancel each other under the mainlobe of the filter mask in the convolution process. There is only efficient sound radiation at the boundary of the plate due to the partial lack of cancellation. In two-dimensions, this corresponds to the well known theory of corner and edge radiation in plates for modes vibrating below the critical frequency. ${ }^{1,2}$ The results also show that the ideal filter of Eq. (21) introduces ringing artifacts due to the infinite impulse response, whereas these artifacts are much less present in the Lanczos filter of Eq. (22). The ringing artifacts introduced are apparent in Figs. 2(e) and 2(f).

This example is reminiscent of well-known analyses ${ }^{2,3}$ where the efficient and inefficient radiation from plates can be seen as a result of the convolution in the wave number domain between a Dirac delta and a sinc function. The Dirac delta results from the modal spatial frequency of the plate, and the sinc function from the finite extent of the plate. This results in a wave number spectrum with two sinc functions centered at $\left( \pm k_{x}\right)$. Above the critical frequency, the main lobes of the sinc functions fall within the radiation circle, resulting in efficient radiation. Below the critical frequency, only some sidelobes of the sinc functions fall within the radiation circle, giving rise to inefficient radiation. With the approach proposed in this paper, this phenomena can be seen directly as a convolution between the space domain representation of the radiation circle and the acoustic field, as described in the foregoing.

\section{EXPERIMENTAL STUDY}

An experimental study has been conducted to examine the validity and applicability of the method proposed in this paper. To date, only simulations of the supersonic intensity on planar radiators have been published in the literature.
The source under study was a baffled steel plate of dimensions $50 \mathrm{~cm} \times 70 \mathrm{~cm}$, and $1 \mathrm{~mm}$ thick, rigidly mounted at the boundaries (see Fig. 3). The plate was driven at $(x=5$, $y=-10) \mathrm{cm}$. The normal vibration velocity of the plate was measured with a Polytec laser vibrometer OFV 056, over a grid of $26 \times 36$ positions. Based on these measurements, an equivalent source method model ${ }^{11,20}$ was used to calculate the sound pressure and sound intensity on the surface of the plate. The equivalent sources were conformal to the measurement positions, retracted $3 \mathrm{~cm}$ behind the plate. To verify the calculation of the pressure, the results were compared to the ones obtained by evaluating the Rayleigh integral via FFT as in Ref. 21 The results from the two methods were nearly identical, with a deviation lower than $5 \%$.

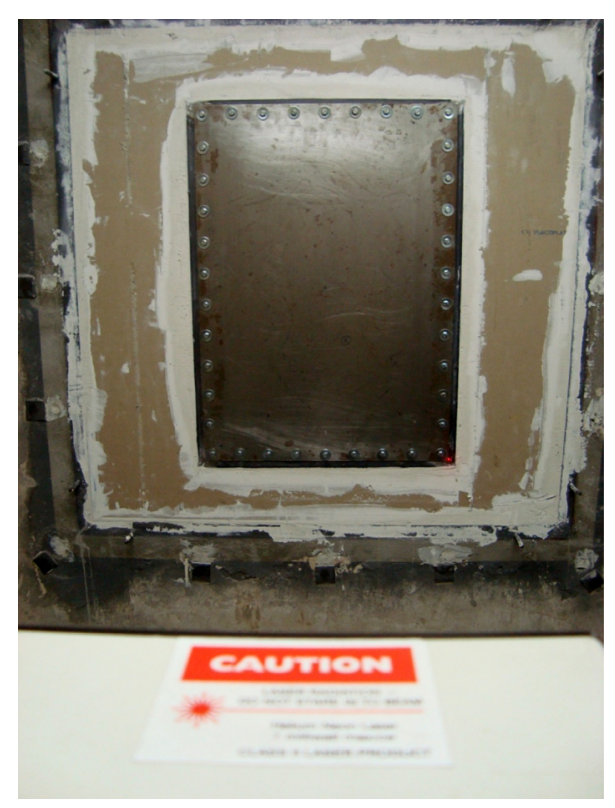

FIG. 3. (Color online) The laser measurements. 
Note that an analogous methodology could be used if instead of measuring the normal velocity of the plate, the sound pressure was measured with a microphone array in the near-field of the source, as in near-field acoustic holography. However, in this study, laser measurements have been used for simplicity and because they provide a clear illustration.

In order to minimize ringing artifacts in the estimation of the supersonic intensity and limit the extent of the filter mask, a windowed version of the ideal filter is proposed. It follows the concept of Lanczos filtering, but it is adapted to the circularly symmetric filter mask, basing the window weights on the mainlobe of a first order Bessel function of the first kind. Therefore, a non-separable radiation kernel is used in which the infinite ideal response is weighted with the mainlobe of a Bessel function. This kernel is better suited for the discrete nature of the practical measurement. It not only minimizes the ringing artifacts, but it also reduces the size of the filter mask, making it computationally more efficient,

$$
h_{w}^{(s)}(\rho)= \begin{cases}\frac{A k}{2 \pi \rho^{2}} J_{1}(k \rho) J_{1}(k \rho / \xi), & \text { for } k \rho<\xi \\ 0 & \text { otherwise }\end{cases}
$$

where $A$ is a normalizing factor of the window. The value of the parameter $\xi$ determines the size of the filter and the number of sidelobes included in the filter mask, and consequently the greater or lesser cut-off slope. The value of $\xi$ could be any positive real number, but it is advantageous when it is one of the zero crossings of the first order Bessel function of the first kind $[\xi=3.8317,7.0156,10.1735,13.3237, \ldots]$. In such case, the ideal infinite filter and the weighting Bessel mainlobe are both zero at the edge of the filter mask, making it continuous and differentiable to second order, providing a smooth cut-off.

In this study, the third zero-crossing of the Bessel function was used, $\xi=10.1735$. Nevertheless, using other values $(\xi=7.0156,13.3237, \ldots)$ does not change the results significantly. The filter is shown in Fig. 4. Comparing it with the ideal filter in Eq. (17), shown in Fig. 1, it is worth noting that the mainlobes of the two filter masks are virtually identical, whereas the sidelobes of the finite filter are soon tapered to zero, limiting its extent and consequently minimizing the characteristic ringing of the ideal filter.

Figure 5 shows the active sound intensity and the supersonic acoustic intensity on the surface of the plate at $125 \mathrm{~Hz}$, which corresponds to a $(2,2)$ modal shape. Note the alternating positive and negative active intensity regions, indicating a circulating flow of energy from one region of the plate into another, giving rise to a very poor far field radiation, as shown by the supersonic intensity map. It should be noted that the wavelength in air is much larger than the dimensions of the plate, and the supersonic intensity just shows a maximum near the driving point, as a source of far field radiation. It is apparent that there is an imaging constraint due to the resolution limit of the wavelength in air compared to the size of the aperture.

Figure 6 shows the active sound intensity and the supersonic intensity on the surface of the plate at $950 \mathrm{~Hz}$, which
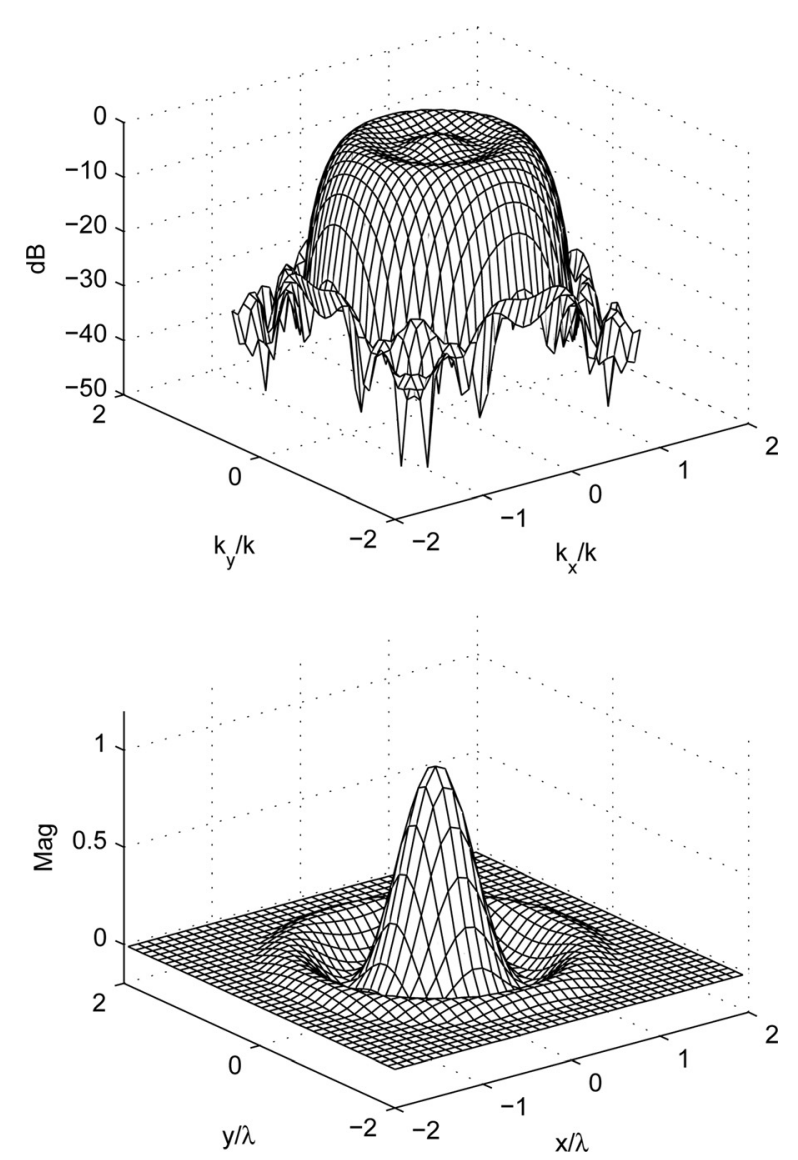

FIG. 4. (Top) Finite radiation filter in the wave number domain $H^{(s)}\left(k_{x}, k_{y}\right)$ and (bottom) the corresponding radiation kernel in the space domain $h_{w}^{(s)}(x, y)$ as in Eq. (23), with $\xi=10.1735$. The overshooting at the pass band is of $\pm 1.2 \mathrm{~dB}$ and the highest sidelobe level in the frequency response is $-30 \mathrm{~dB}$.

corresponds to a $(4,10)$ modal shape. This frequency is also below coincidence, and a very reactive sound field is found. In this case, there are multiple regions with alternating positive and negative intensities, making it more difficult to identify and quantify how are they contributing to the net power output of the source. However, the supersonic intensity map indicates that the main radiation is from the corners of the plate, where there is a partial lack of cancellation at the edges. This result agrees with the well-known theory of corner and edge radiation from plates.

Figure 7 shows the active intensity and the supersonic intensity on the surface of the plate at $1135 \mathrm{~Hz}$. At this frequency both corner and edge modes seem to be excited. There is a very high modal density, and the vibration pattern cannot be associated with an individual mode shape. The supersonic intensity map shows that there is in fact effective sound radiation from the corners and edges of the plate into the far field. Note that in all three cases (Figs. 5-7) the supersonic intensity level is significantly lower than the active sound intensity due to the circulation of energy occurring below coincidence.

Figure 8 shows the supersonic intensity of the plate at 950 and $1135 \mathrm{~Hz}$ calculated using FFT based convolution. The results are closely similar to those in Figs. 6 and 7, obtained via direct convolution with the finite filter in 

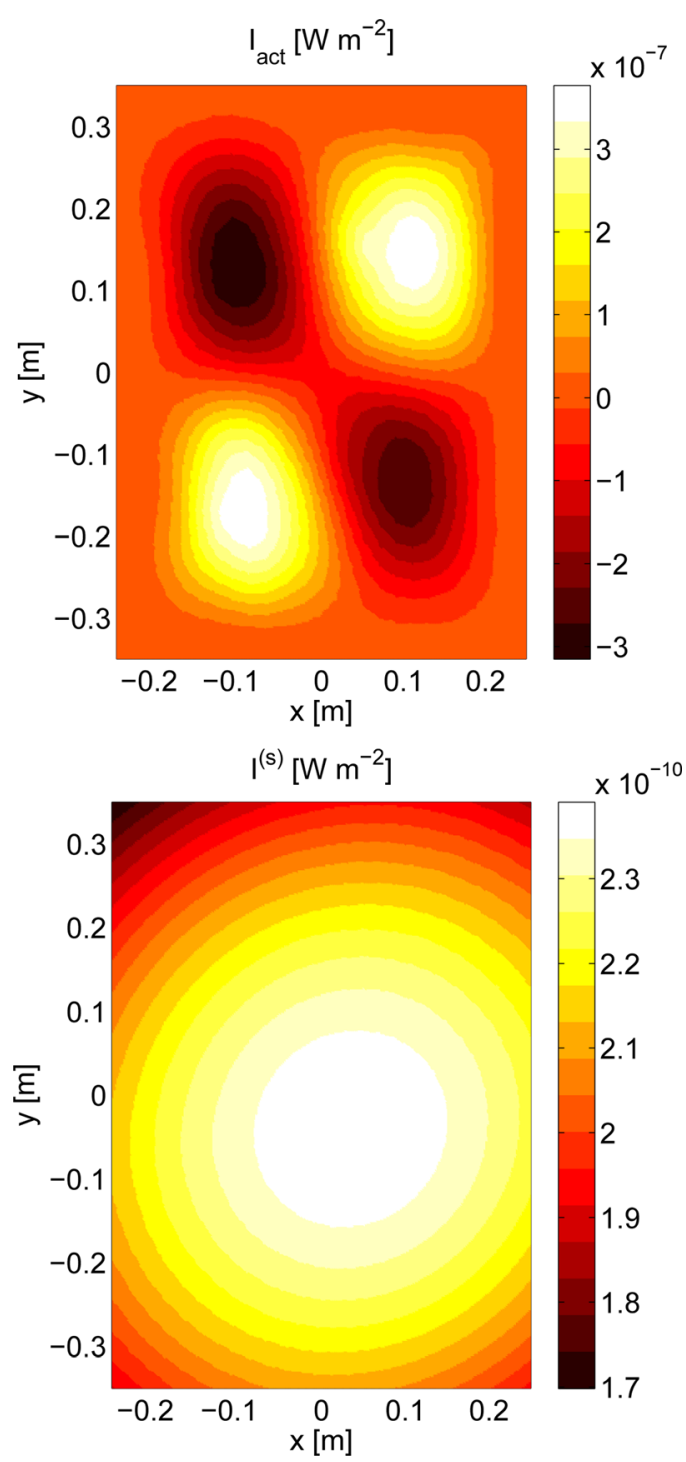

FIG. 5. (Color online) (Top) Active sound intensity on the source plane and (bottom) the supersonic sound intensity at $125 \mathrm{~Hz}$. Note the negative active sound intensity.

Eq. (23), indicating the underlying equivalence between the two methodologies.

It has been shown that the supersonic intensity represents the fraction of acoustic energy that contributes to the total power output from the source, in other words, that there is conservation of power. ${ }^{9,10}$ In this experimental study, the total power radiated by the source has been calculated from the active sound intensity, as well as from the estimated supersonic intensity. At $950 \mathrm{~Hz}$, the power calculated from the active intensity is $1.5 \times 10^{-10} \mathrm{~W}$ and from the supersonic intensity it is $1 \times 10^{-10} \mathrm{~W}(\sim 1.7 \mathrm{~dB}$ deviation $)$. At $1135 \mathrm{~Hz}$, the power calculated from the active intensity is $2.5 \times 10^{-9} \mathrm{~W}$, and from the supersonic intensity it is $1.5 \times 10^{-9} \mathrm{~W}(\sim 2 \mathrm{~dB}$ deviation $)$.

The sound power calculated from the supersonic intensity is somewhat underestimated due to the truncation introduced by the finite measurement aperture. The underestimation of the sound power is more pronounced in the low frequency range, where the acoustical wavelength, which determines the width of the radiation kernel, is much larger than the aperture. In the present experiment, at $127 \mathrm{~Hz}$, there
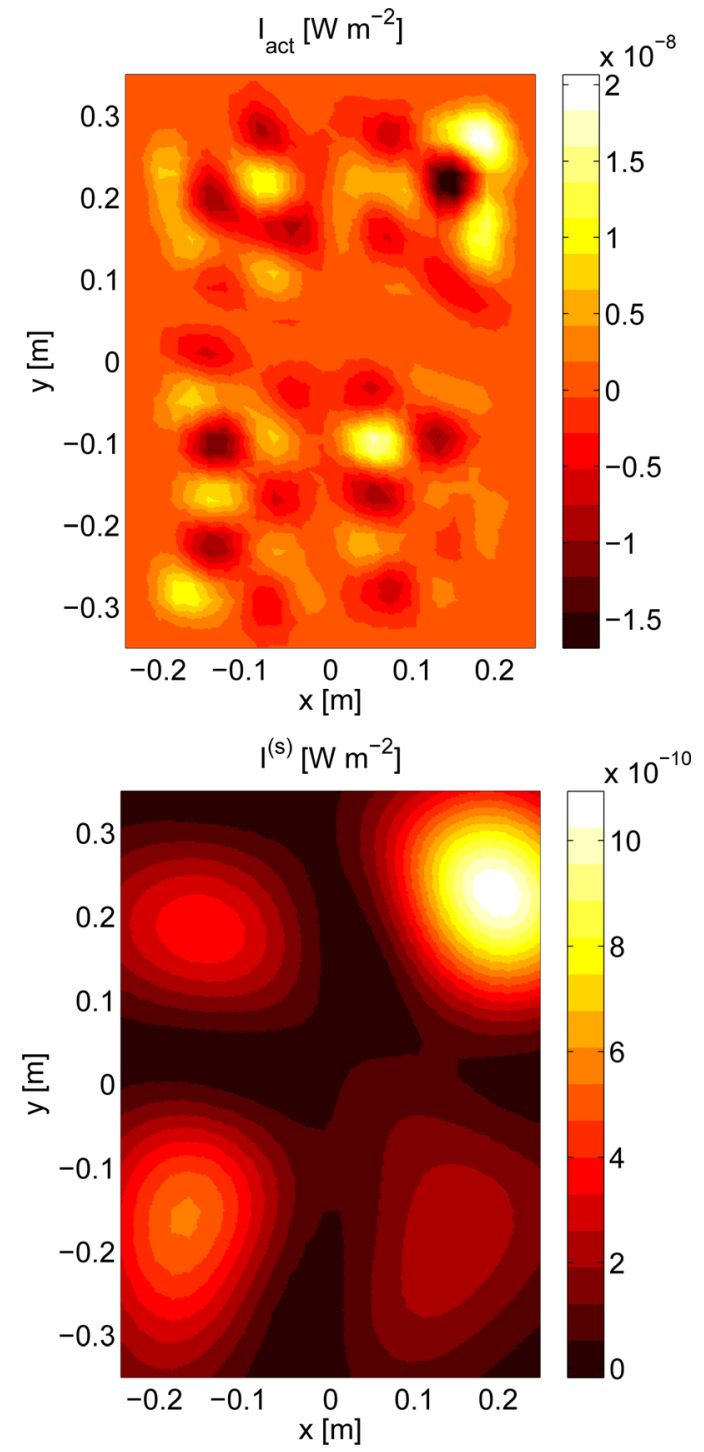

FIG. 6. (Color online) (Top) Active sound intensity on the source plane and supersonic sound intensity (bottom) at $950 \mathrm{~Hz}$.

was an underestimation of the power of about $8 \mathrm{~dB}$. However, if the size of the measurement aperture is increased to $82 \times 102 \mathrm{~cm}^{2}$ (this was done by zero-padding the measured normal velocity and calculating the corresponding sound pressure over a larger aperture), the underestimation at $127 \mathrm{~Hz}$ is reduced to $4 \mathrm{~dB}$, whereas at 950 and $1135 \mathrm{~Hz}$ it is less than $1 \mathrm{~dB}$. Eventually, the estimation always converges to the correct radiated power by sufficiently extending the measurement aperture, also at lower frequencies, due to the conservation of power.

Lastly, regarding the calculation method, the total power estimated via either direct convolution or FFT processing was practically the same, within $\pm 0.25 \mathrm{~dB}$. On the whole, the experimental results confirm that the supersonic intensity is a meaningful measure of the net far field output of the source.

\section{DISCUSSION}

The formulation presented in this paper seems to be convenient for applications where the explicit transformation of sound fields into the $k$-space domain is not required. This is the 

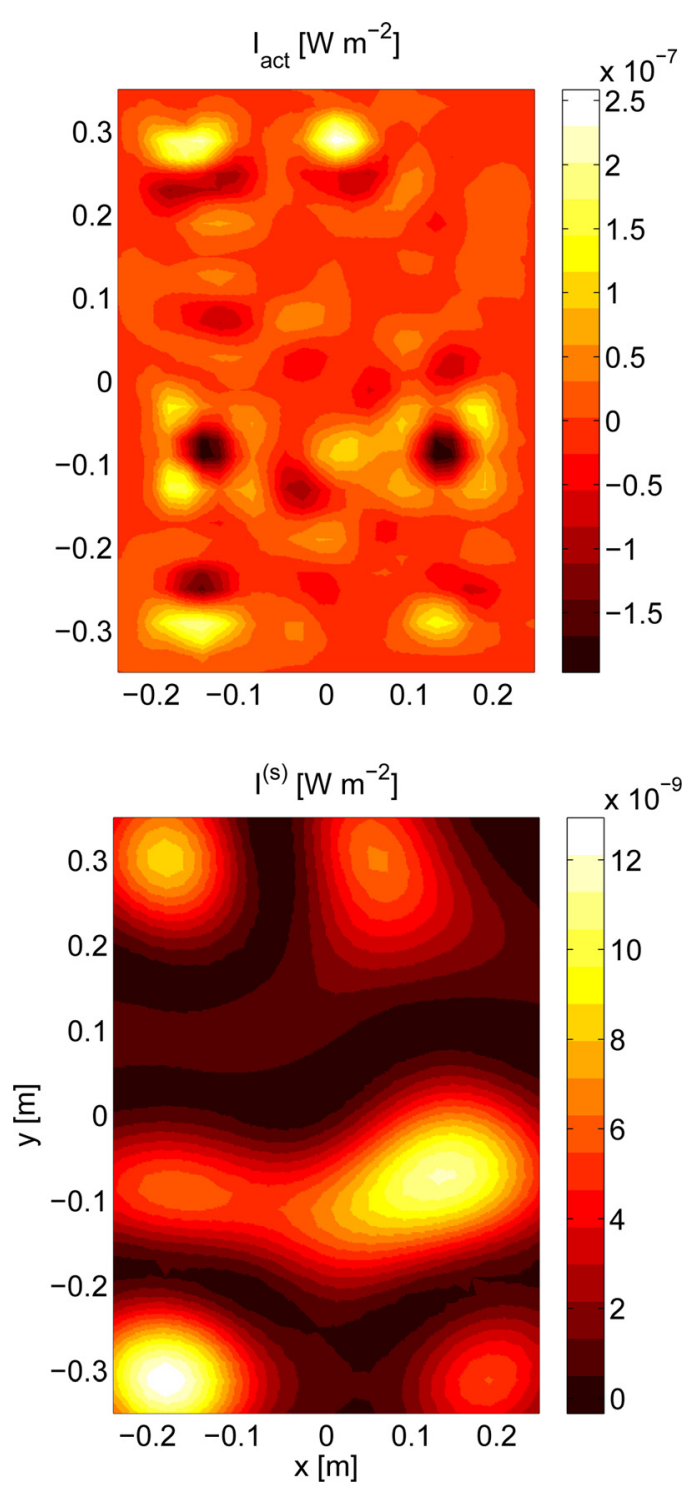

FIG. 7. (Color online) (Top) Active sound intensity on the source plane and (bottom) the supersonic intensity at $1135 \mathrm{~Hz}$.

case for many of the existing holography techniques, where the direct formulation proposed here could be applied directly after the reconstruction of the acoustic field. The methodology could also be applied directly to measurements or to numerical calculations of vibrating sources, in order to provide an estimation of the effective radiation of the source into the far field.

The Fourier based convolution and the direct convolution operations are closely related to each other, yielding similar results, although there are some fundamental differences. Most notably, the Fourier based convolution assumes periodicity of the signals, and therefore proper zero padding is required (to at least the size of the measurement positions plus the filter mask), to avoid possible errors. The direct convolution does not assume periodicity, and it is a simple operation that can be more accurate on the boundaries of the data regardless of the size of the measurement and the filter mask.

Regarding the computational complexity of the two implementations, given an array of size $N \times N$, a radiation filter mask of dimensions $L \times L$, and an output array of $M \times M$, where $M=N+L-1$, the number of operations
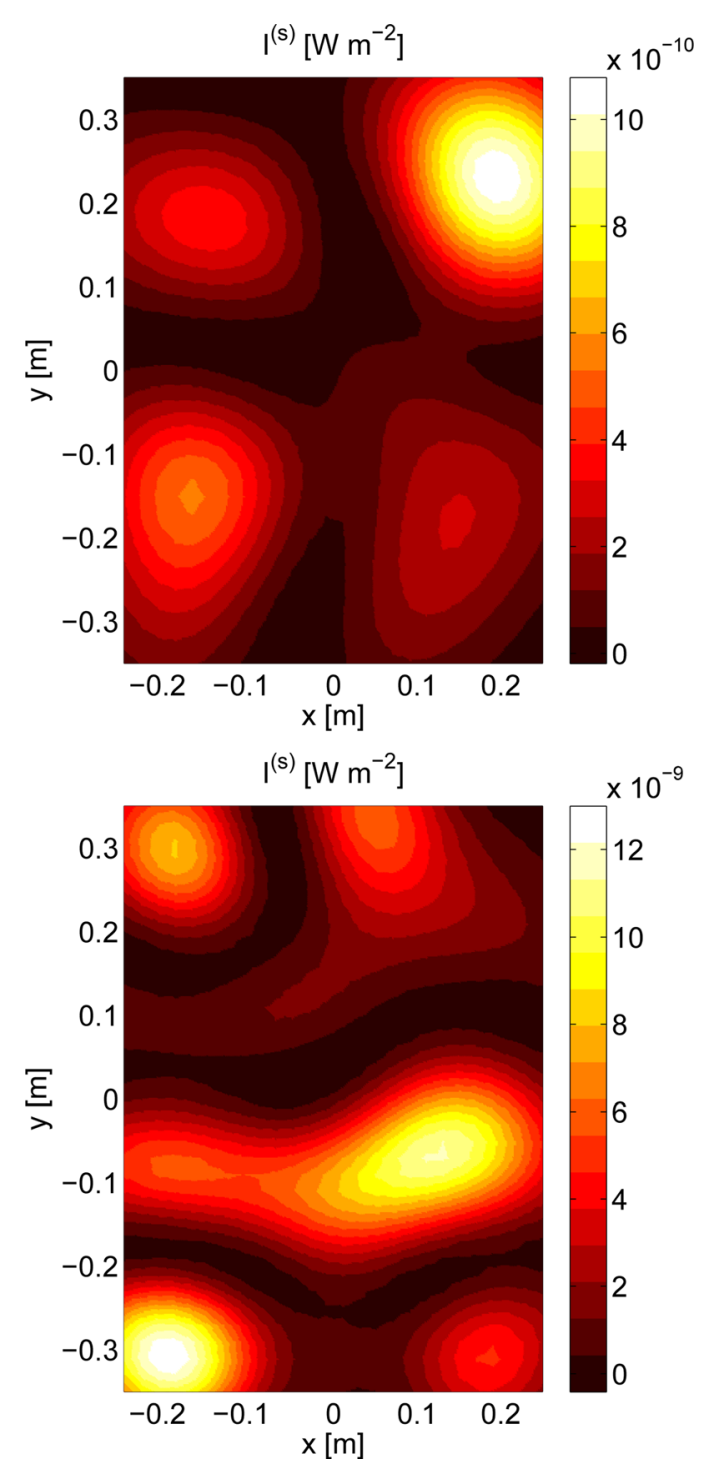

FIG. 8. (Color online) Supersonic intensity (as in Figs. 6 and 7), calculated using FFT-based filtering. (Top) $950 \mathrm{~Hz}$; (bottom) $1350 \mathrm{~Hz}$.

required in a two-dimensional convolution is of order $M^{2} N^{2}$. The number of operations using FFT has a lower bound of order $M^{2}\left(1+2 \log _{2}\left(M^{2}\right)\right) \cdot{ }^{16,22}$ In some disciplines, such as image processing, the input vectors or matrices are typically very large, containing at least several millions of elements. Therefore, the FFT implementation of the filtering process can save a tremendous computation effort. However, in the case of array acoustics, where typically the input vectors are of a few hundred points or less, there is not a clear computational basis for preferring an FFT implementation, because the complexity of the operation is small in any case. Furthermore, for few points, the direct convolution can be computationally lighter.

It has been shown in the foregoing how the sharp cutoff in the wave number domain due to the ideal filter introduces ringing artifacts via the Gibbs phenomenon. Although the sharp ideal cut off is theoretically rigorous, it poses some difficulties in practice. Therefore, it is convenient to use instead a finite filter that is suited for the practical implementation. In the wave number domain this can be done by choosing an appropriate lowpass FIR filter 
(Butterworth, etc.). ${ }^{22}$ Alternatively, the space domain formulation proposed in this paper makes it possible to design the desired filter mask by means of the straightforward and well-known windowing method. ${ }^{23}$

It is not the purpose of this paper to make a comparison between the many available filters. Nevertheless, a nonseparable circularly symmetric filter suited to the problem has been proposed. It provides a good compromise between sharp cut-off, and low ringing artifacts.

\section{CONCLUSIONS}

In this paper, the concept of supersonic acoustic intensity has been examined and formulated as a direct filtering operation in space domain. The method makes it possible to identify the regions of a source that radiate into the far field and to estimate how much of the acoustic energy flow propagates to the far field, contributing to the net power output of the source.

A numerical example as well as an experimental study have served to illustrate the method and examine its advantages and limitations. The method is appealing due to its simplicity and the fact that it does not require transformations into the wave number domain. Hence, it can be useful in the general case for applications in which the Fourier transformation of sound fields is not required. Ultimately, the formulation presented in this paper contributes to an alternative, yet equivalent description of the near field and far field radiation from acoustic sources.

${ }^{1}$ G. Maidanik, "Response of ribbed panels to reverberant acoustic fields," J. Acoust. Soc. Am. 34, 809-826 (1962).

${ }^{2}$ L. Cremer, M. Heckl, and E. E. Ungar, Structure-Borne Sound (SpringerVerlag, New York, 1973), Chap. 6.

${ }^{3}$ F. J. Fahy, Sound and Structural Vibration, 2nd ed. (Academic Press, London, 1987), Chap. 3.

${ }^{4} \mathrm{~V}$. Borgiotti, "The power radiated by a vibrating body in an acoustic fluid and its determination from boundary measurements," J. Acoust. Soc. Am. 88, 1884-1893 (1990).
${ }^{5}$ D. M. Photiadis, "The relationship of singular value decomposition to wave-vector filtering in sound radiation problems," J. Acoust. Soc. Am. 88, 1152-1159 (1990).

${ }^{6}$ R. A. Magalhaes and M. B. S. Tenenbaum, "Supersonic acoustic intensity for arbitrarily shaped sources," Acta. Acust. Acust. 92, 189-201 (2006).

${ }^{7}$ J. D. Maynard, E. G. Williams, and Y. Lee, "Nearfield acoustic holography I: Theory of generalized holography and the development of NAH," J. Acoust. Soc. Am, 78, 1395-1413 (1985).

${ }^{8}$ E. G. Williams, Fourier Acoustics: Sound Radiation and Nearfield Acoustic Holography (Academic Press, San Diego, 1999), Chaps. 2-3.

${ }^{9}$ E. G. Williams, "Supersonic acoustic intensity," J. Acoust. Soc. Am. 97, 121-127 (1995).

${ }^{10}$ E. G. Williams, "Supersonic acoustic intensity on planar sources," J. Acoust. Soc. Am. 104, 2845-2850 (1998).

${ }^{11}$ A. Sarkissian, "Method of superposition applied to patch near-field acoustic holography," J. Acoust. Soc. Am. 118, 671-678 (2005).

${ }^{12} \mathrm{R}$. Bai, "Application of BEM (boundary element method)-based acoustic holography to radiation analysis of sound sources with arbitrarily shaped geometries," J. Acoust. Soc. Am. 92, 533-549 (1992).

${ }^{13} \mathrm{~J}$. Hald, "Basic theory and properties of statistically optimized near-field acoustical holography," J. Acoust. Soc. Am. 125, 2105-2120 (2009).

${ }^{14}$ E. Fernandez-Grande and F. Jacobsen, "Supersonic acoustic intensity with statistically optimized near-field acoustic holography," in Proceedings of Inter-Noise 2011 (CD-Rom), Osaka, Japan (September 4-7, 2011).

${ }^{15}$ M. Abramowitz and I. A. Stegun, Handbook of Mathematical Functions (National Bureau of Standards, Washington, D.C., 1964), Chap. 9.

${ }^{16}$ W. K. Pratt, Digital Image Processing, 3rd ed. (Wiley-Interscience, New York, 2001), Chap. 7.

${ }^{17}$ W. K. Pratt, "Vector space formulation of two-dimensional signal processing operations," Comput. Vision Graph. 4, 1-24 (1975).

${ }^{18}$ C. E. Duchon, "Lanczos filtering in one and two dimensions," J. Appl. Meteorol. 18, 1016-1022 (1979).

${ }^{19}$ W. Burger and M. J. Burge, Principles of Digital Image Processing: Core Algorithms, 1st ed. (Springer-Verlag, London, 2009), Chap. 10.

${ }^{20}$ G. H. Koopmann, L. Song, and J. B. Fahnline, "A method for computing acoustic fields based on the principle of wave superposition," J. Acoust. Soc. Am. 86, 2433-2438 (1989).

${ }^{21}$ E. G. Williams and J. D. Maynard, "Numerical evaluation of the Rayleigh integral for planar radiators using the FFT," J. Acoust. Soc. Am. 72, 2020-2030 (1982).

${ }^{22}$ R. C. Gonzalez and R. E. Woods, Digital Image Processing, 3rd ed. (Prentice Hall, Upper Saddle River, NJ, 2008), Chap. 4.

${ }^{23}$ A. V. Oppenheim and R. W. Schafer, Discrete-Time Signal Processing, international edition (Prentice-Hall, Upper Saddle River, NJ, 1989), Chap. 7. 